\title{
A Smart Pill Box to Remind of Consumption using loT
}

\author{
Shilpa Khedkar \\ M.E.S. College of Engineering \\ Doreen Charles \\ M.E.S. College of Engineering
}

\author{
Sagarika Deshpande \\ M.E.S. College of Engineering
}

\author{
Manasi Choudhari \\ M.E.S. College of Engineering
}

\author{
Sarish Shaikh \\ M.E.S College of Engineering
}

\begin{abstract}
The progress in medical technologies is one of the main contributions to the aging population. Most of the elders have chronic diseases and they need to take medications for a prolonged period of time in order to stabilize their conditions. So medication safety for the elderly is extremely vital. The most commonly encountered situations of drug abuse are excessive drug usage and disobeying the medication instructions. We have developed a device called "smart pillbox" to help the aging population to consume the right medication at the appropriate time according to their requirements. This system improves the existing system by alerting the user at the particular time again by setting the count each time and the order for the particular pill is sent by the system automatically to medical shop through message gateway and email.
\end{abstract}

\section{General Terms}

Internet of Things, Medication Safety.

\section{Keywords}

Smart Pillbox, Medication Reminders, IoT, Order for pills, Message Gateway and Email, Arduino Wemos D1R1.

\section{INTRODUCTION}

Poor medication adherence is one of the major causes of illness and of treatment failure. The classical practice of dispensing medication to a patient has allowed the patient to take the medication by himself, or delegate those responsibilities to a keeper or a doctor. The supervision by nurses and doctors is often expensive and unrealistic for the administration of medicine within home. Forgetting to take medication or taking wrong dosages is common in elderly patients who frequently are lonely and lose track of time [6]. Nowadays there are systems like scheduled alarm clocks or apps dedicated to schedule and notify medication's time in

cell phones. Also there are pill organizers commonly used by patients to remind themselves of the dosages. The disadvantages of those systems are that the pills cannot be stored for a long time and it doesn't have an alarm system. The connectivity of sensors and other healthcare devices (loT) play an important role in care of patients, because it allows getting access in real-time of medical information. Thus, the study and development of an effective Healthcare/loT gateway could be crucial in patient care.[1]

In this paper, we propose a new way of reminding and taking dosages at the right time by using new technologies linked to free hardware and software, with a low cost that does not have limitations on licenses and functions which allows the organization of several medication schedules that health disorders used to present in elderly need.

\section{RELATED WORK}

The approach related to the design of AT device [1] gives a new choice in taking medication dosages. It gives a programmable alarm system with an automatic opening and closing system, an interactive user interface and a notification system through GSM network. A pillbox called a MedTracker [2] monitoring of medication adherence on a continuous basis. It gives more detailed information about non-adherence and medication errors, and the familiar interface of a 7-day drug store pillbox. A smart pillbox equipped with a camera and based on the medicine bag [3] concept. The matrix barcode printed on the medicine bags is used to interact with the pillbox in order to perform pill remind and confirm functions. An intelligent pill box (IPB)[4] is based on the medicine bag [3][4]system, and the IPB sends a medicine bag out of the box at the appropriate time. If the patient does not take the medicine bag away, the IPB would notify the caregivers via Skype. The IPB system improves the interactivity between patient and caregivers, but it works well only if an internet connection is available. A pillbox [5]based on an MCS-51 micro-controller can send out medicine using a stepper motor at a scheduled time, but there was no provision to record the time when the patient actually took the medicine.

\section{PROPOSED SYSTEM}

In day to day life, people have trouble remembering the pills they need to take from the medicine bag. Multiple times the problem is that the time required to take the medicine is not printed on the box of medicine or they can't read English. People also have a habit to forget to take the pills. Due to this, some medicines expire.

In order to reduce the responsibility of family members, the proposed pill box is of great help by adding required medicine to the pillbox and setting the pill time for that particular medicine. The Real-time clock is used to identify the pill time. If the system time matches with pill time the buzzer starts to ring until the push button is pressed after taking the pills. Figure 2 gives the architecture of the proposed system. When the push button is pressed the buzzer stops and the pills required to be taken at that time comes out to the user to avoid confusion among medicines. Multiple times a user requires more than one pill of same the medicine or more than one people are using the same system. So it is required to update the count of number of pills removed by the user. If pills remain less, the purchase order is sent to the medical shop by using message gateway and an email. Figure 1 gives the flow of the proposed system.

The user uses the android application to set the pill timings, which pills need to be taken, dosage of the pills. The application also has functionality to give a reminder to take a syrup which is also set by the user. 


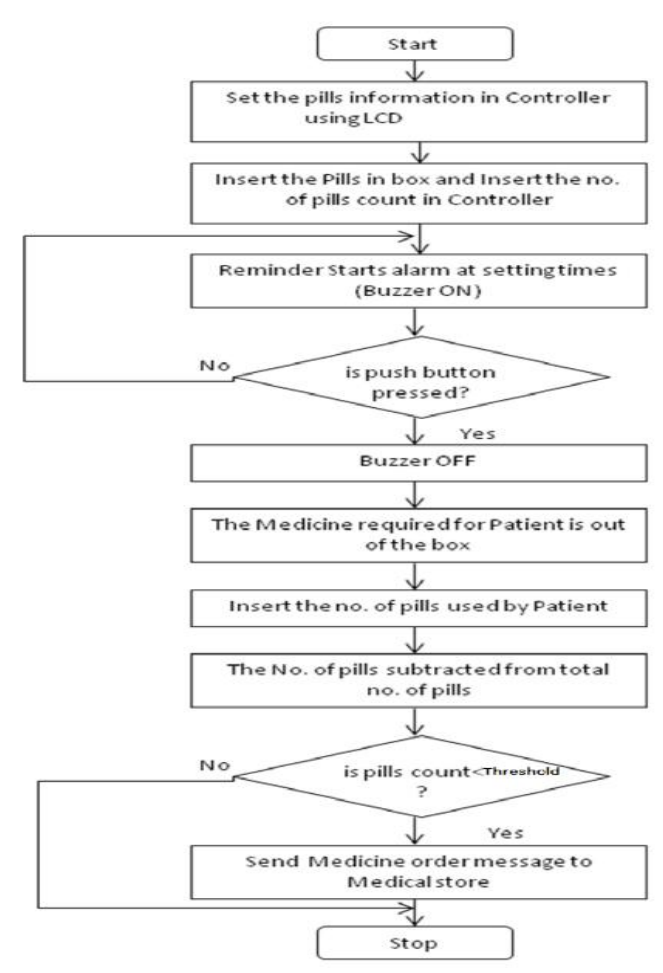

Fig-1: Flowchart of the System

\section{ARCHITECTURE}

1. Set the time for pills: Set the pill time for required medicine by using input system. We can set different time for different pills. If the more than one pill is required at a time, give the box nos. to the system to get required pills. We also set the no. of pills we are inserting in system.

2. Compare the time using real time clock: The real time clock gives continuous time as an output. Monitor the time continuously using Real time clock to identify the pill time. If the system time matches with pill time, the system shows that that it is time to take pill.

3. Alert the user to take pills: It is necessary to alert the user to take pills at particular time. When the system time match with pill time, the buzzer start continuously until the push button is not pressed. When the push button pressed, the buzzer stops and the pills required to take at that time comes out to user to avoid confusion among medicines.

4. Get the feedback about pills from user: As pills removed by user, it is necessary to put the no. of pills removed by user. Multiple times a user required more than one pills of same medicine or more than one person are using same system. So it is required that the no. of pills removed by user.

5. Send purchase order to medical shop: The system counts no. of pills in the system by using the total no. of pills and the pills used by patient. When the no. of pills remains less, the purchase order sends automatically to medical shop.

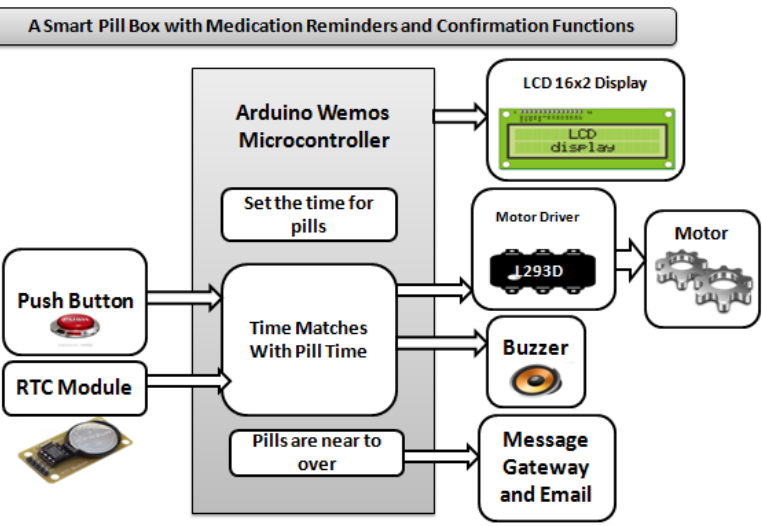

Fig-2: Architecture of the pillbox

\section{RESULTS}

The working experimental setup of the proposed system is given in below figure which gives the alert at the time of pill taken. The Pill box rotates and pill which is to be taken can be removed from the box.

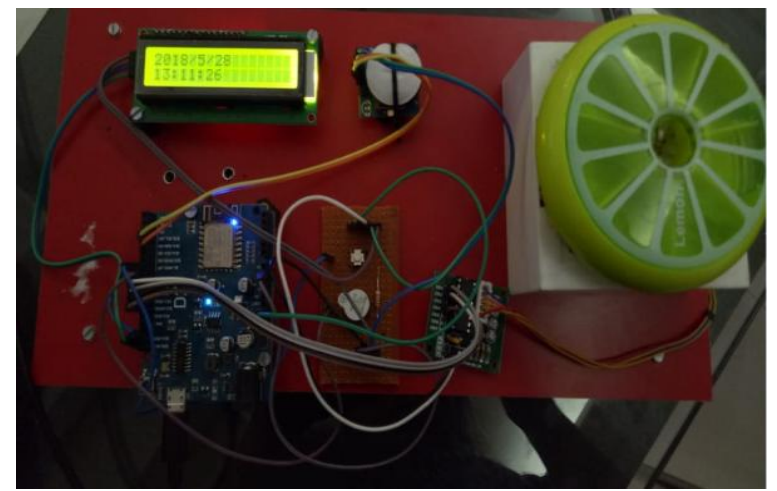

Fig-3: Experimental Setup

For using this system admin have to register the patient with name, age etc as shown in Figure 4.

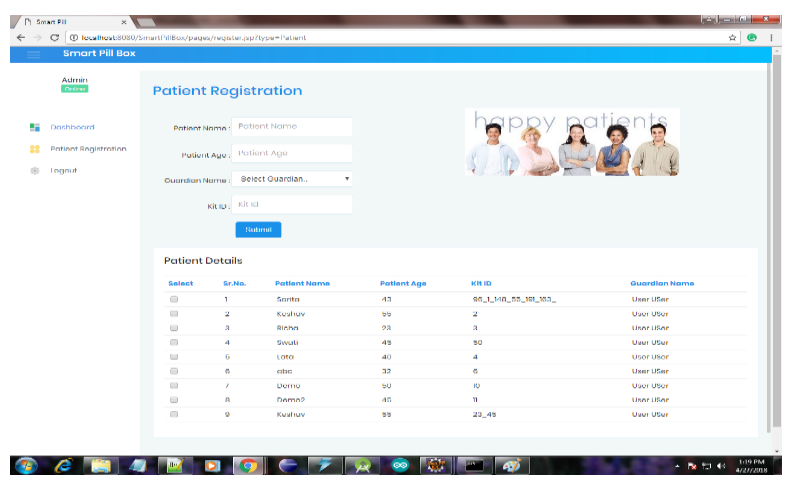

Fig-4: Patient Registration Page

After registration guardian can add the medicine with name and dosage and set the timing for that pill. 


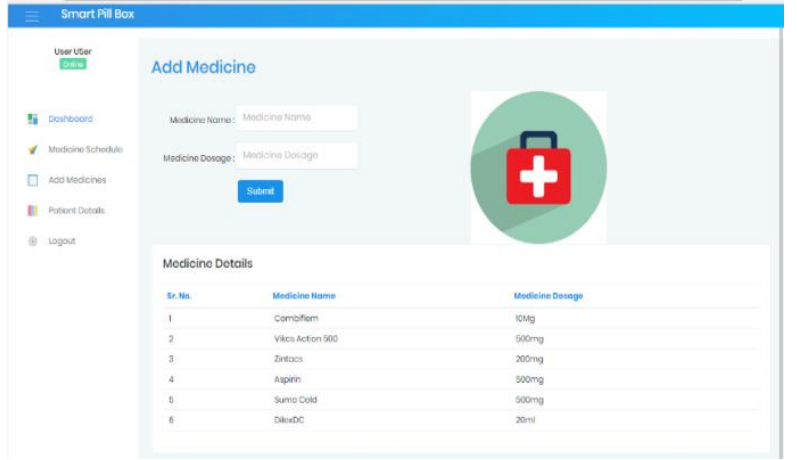

Fig-5: Add Medicine

The time is set for the particular medicine with the count of the pills to be taken.

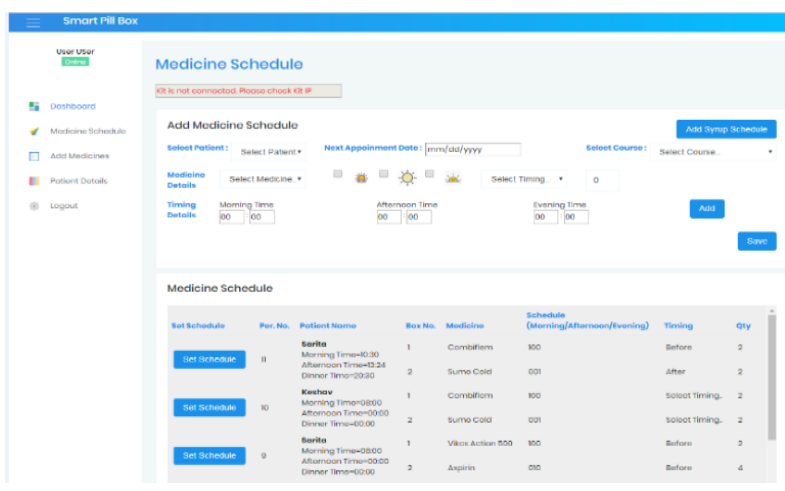

Fig-6: Medicine Schedule

We can also get the medicine details to check if the patient has taken the medicine or not.

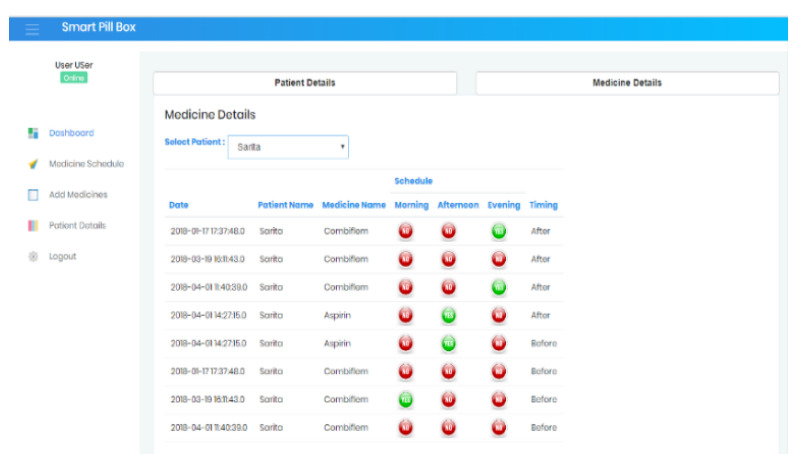

Fig-7: Medicine Details

\section{CONCLUSION}

Elderly people play an important role in the society. They are part of the priority group of healthcare. Therefore it is necessary to create new devices using the emerging technology in order to improve their lives quality. Based on open source solutions, a new alternative to remind medicine dosages is proposed. Arduino Wemos as main controller works totally right and give many other opportunities to develop. The objective of creating a device that allows the organization of several medication schedules, automatic opening system and an effective notification system, is reached. This system reduces family member's responsibility towards ensuring the correct and timely consumption of medicines by alerting the user to get the pill at the particular time.

\section{REFERENCES}

[1] Juan Marcelo Parra 1, Wilson Valdez, Andrea Guevara, Priscila Cedillo,Jose Ortiz Segarra,"INTELLIGENT PILLBOX: AUTOMATIC AND PROGRAMMABLE ASSISTIVE TECHNOLOGY DEVICE",Proceedings of the 1ASTED International Conference Biomedical Engineering (BioMed 2017).

[2] "An Electronic Pillbox for Continuous Monitoring of Medication Adherence",Tamara. L. Hayes, Member, IEEE, John M. Hunt, Member IEEE,28th IEEE EMBS Annual International Conference New York City, USA, Aug 30-Sept 3, 2006.

[3] Huai-Kuei Wu1, Member, IEEE, Chi-Ming Wong, PangHsing Liu, Sheng-Po Peng, Xun-Cong Wang, Chih-Hi Lin and Kuan-Hui Tu"A Smart Pill Box with Remind and Consumption Confirmation Functions", 2015 IEEE 4th Global Conference on Consumer Electronics (GCCE).

[4] S.-C. Huang, H.-Y. Chang,Y.-C. Jhu and G.-Y. Chen, "The intelligent pill box-design and implementation," in proceedings of the IEEE International Conference on Consumer Electronics, May 26-28, Taiwan.

[5] G H.-W. Kuo, "Research and Implementation of Intelligent MedicalBox," M.S.thesis, Department of Electrical Engineering, I-Shou University, Kaohsiung, TW, 2009.

[6] J. H. Brown, P. E. N. Knowles, F. Booth, and H. C. Post, "United States Patent" 909628, 1980. 